\title{
SMARCA2/BRM-Deficient Undifferentiated/Rhabdoid Carcinoma of Unknown Primary Site
}

\author{
Yasutaka Tono $^{a}$ Koushi Sukeno ${ }^{a}$ Akira Tsunoda $^{a}$ Kanako Saito $^{a}$ \\ Yoshiki Yamashita $^{a}$ Miki Usui $^{\text {b }}$ Katsunori Uchida ${ }^{\text {b }}$ Hiroshi Imai $^{\text {b }}$ \\ Toshiro Mizuno $^{a} \quad$ Isao Tawara ${ }^{a, c}$ \\ aDepartment of Medical Oncology, Mie University Hospital, Tsu, Japan; bPathology Division, \\ Mie University Hospital, Tsu, Japan; 'Department of Hematology and Oncology, Mie \\ University Graduate School of Medicine, Tsu, Japan
}

\section{Keywords}

Cancer of unknown primary site - Undifferentiated $\cdot$ SWItch/sucrose nonfermentable

SMARCA2 - BRM

\begin{abstract}
Undifferentiated neoplasms of unknown primary sites are rare. It is difficult to identify their characteristics and determine the appropriate chemotherapy regimen to be used. Undifferentiated/rhabdoid carcinoma is reportedly associated with loss of SWI/SNF chromatin remodeling complexes, such as observed in SMARCA4-deficient tumors. However, little is known about SMARCA2/BRM-deficient tumors. A 48-year-old man presented with low back pain. Computed tomography $(\mathrm{CT})$ revealed intraperitoneal lymph nodes and multiple bone metastases that invaded the thoracic and lumbar spinal canals. The primary tumor was not identified despite the standard diagnostic methods being used. CT-guided needle biopsy of right iliac bone metastasis showed that the tumor had an undifferentiated/rhabdoid morphology. Immunostaining revealed that the tumor was SMARCA2/BRM-deficient despite both SMARCB1/ INI1 and SMARCA4/BRG being retained. We found no genomic alterations during domestic next-generation sequencing panel profiling, which can identify 114 genes. Thus, he was diagnosed with SMARCA2/BRM-deficient undifferentiated/rhabdoid carcinoma of an unknown primary site with multiple bone metastases and intraperitoneal lymph node metastasis. We administered radiotherapy to the thoracic and lumbar spine to improve cord compression, and carboplatin (CBDCA) and paclitaxel regimen was chosen as first-line chemotherapy, but this was discontinued due to an anaphylactic shock. We then selected the CBDCA and gemcitabine regimens; however, the patient did not continuously receive the regimen due to myelosuppression.
\end{abstract}

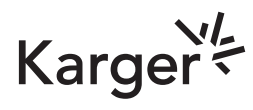


Radiation therapy effectively relieves pain and cord compression. To our knowledge, this is the first reported case of SMARCA2/BRM-deficient undifferentiated/rhabdoid carcinoma of an unknown primary site. Further studies are needed to improve SWI/SNF-deficient tumor identification methods.

\section{Introduction}

Cancer of unknown primary site (CUP) is a histologically confirmed type of metastatic cancer for which clinicians cannot detect a primary lesion despite using a standard diagnostic approach. Undifferentiated neoplasms of unknown primary sites comprise approximately less than $5 \%$ of all CUP [1], and no appropriate chemotherapy regimen is available for their treatment. Recently, gene expression profiling has been useful to confirm some frequent alterations and support novel therapeutic approaches for the driver mutation in various cancers. In CUP, identification of molecular characteristics by a multiple gene assay triggers to assess the primary lesion and find appropriate molecular target agents.

In undifferentiated neoplasms, tumors with undifferentiated/rhabdoid morphology on pathological findings have been associated with abnormalities in SWItch/sucrose nonfermentable (SWI/SNF) chromatin remodeling complexes [2], which comprise multiple subunits (e.g., a BRG-encoding gene in SMARCA4, a BRM-encoding gene in SMARCA2, an INI1-encoding gene in SMARCB1, and ARID1A) [3].SWI/SNF-deficient tumors include the following: SMARCA4-deficient thoracic sarcoma [4]; small-cell carcinoma of the ovary hypercalcemic type, which is deficient in SMARCA4 [5]; and malignant rhabdoid tumor, which is deficient in SMARCB1 [6]. However, little is known about SMARCA2/BRM-deficient tumors. In the present study, we report a case of SMARCA2/BRM-deficient undifferentiated/rhabdoid carcinoma of an unknown primary site.

\section{Case Presentation}

A 48-year-old man presented to a local hospital with complaints of low back pain. His medical history included hypertension, diabetes mellitus, and bronchial asthma. His hypertension was controlled with irbesartan $100 \mathrm{mg}$, bisoprolol $2.5 \mathrm{mg}$, amlodipine $5 \mathrm{mg}$, and diabetes mellitus with tofogliflozin $20 \mathrm{mg}$. Furthermore, his family medical history revealed no history of any malignant tumors. Computed tomography (CT) revealed swollen intraperitoneal lymph nodes and multiple bone masses in the spine, ribs, right scapula, left clavicle, sternum, bilateral ilia, left pubic bone, left ischium, and left femur. Spine magnetic resonance imaging showed invasion into the spinal canal at the Th4 and Th11 levels (shown in Fig. 1). The primary lesion was not found on CT, and upper gastrointestinal endoscopy and colonoscopy also did not reveal the primary tumor. He was suspected of having CUP and then referred to our hospital after 2 months of his first visit to the previous hospital. CT-guided needle biopsy of the right iliac bone metastasis was performed, and histopathological examination revealed diffuse growth of tumor cells with a high nuclear-cytoplasmic ratio. Moreover, some tumor cells with unevenly distributed nuclei were also observed. Scattered mitotic images of tumor cells were observed, and some tumor cells with large nuclei were included. Importantly, some tumor cells had rhabdoid features (shown in Fig. 2a, b). Immunohistochemistry revealed AE1/AE3, CK7, CK20 (focal), vimentin, and GATA3 positive markers but was negative for CDX-2, LCA, ALK, MUM1, HMB45, uroplakin II, and p63. Given these results, the pathologist ruled out urothelial

\section{Karger's}




\section{Case Reports in Oncology}

\begin{tabular}{l|l}
\hline Case Rep Oncol 2022;15:163-169 \\
\hline DOI: 10.1159/000521632 & $\begin{array}{l}\text { ○ 2022 The Author(s). Published by S. Karger AG, Basel } \\
\text { www.karger.com/cro }\end{array}$ \\
\hline
\end{tabular}

Tono et al.: SMARCA2/BRM-Deficient Carcinoma of Unknown Primary Site

Fig. 1. STIR mode of the spine MRI showing the cord compressions in the Th4 and Th11 levels (arrows). STIR, short T1 inversion recovery; MRI, magnetic resonance imaging.
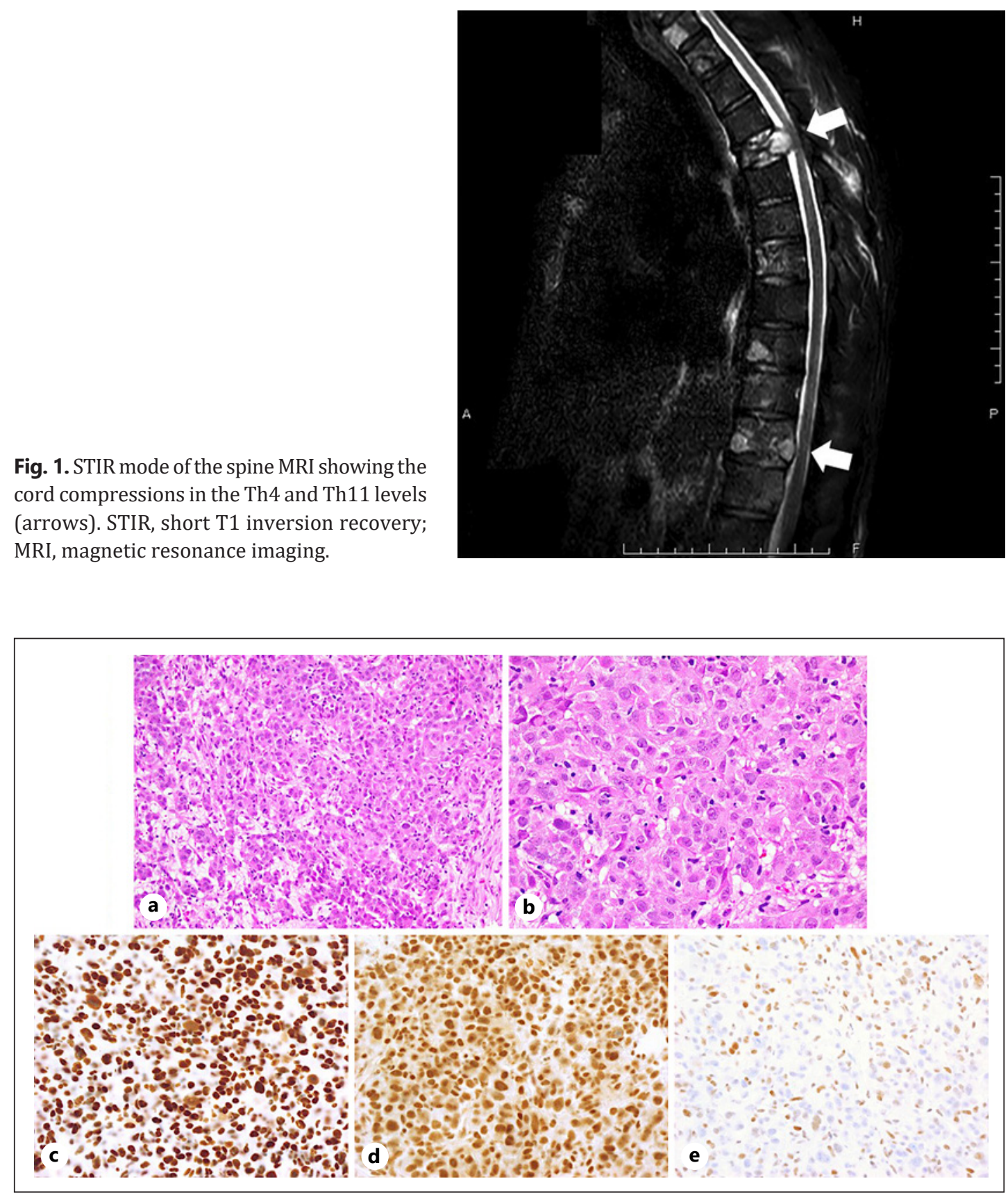

Fig. 2. HE stains and IHC of histopathology. Histopathology of biopsy for his right iliac bone metastasis (a, b); HE stains (c, d); IHC positive for SMARCB1 (INI1) and SMARCA4 (BRG1); IHC negative for SMARCA2 (BRM) (e).

carcinoma, sarcoma, malignant lymphoma, and malignant melanoma. Additional immune staining showed that SMARCB1 (INI1) and SMARCA4 (BRG1) were retained (shown in Fig. 2c, d), but SMARCA2 (BRM) expression was lost (shown in Fig. 2e). We used the OncoGuide ${ }^{\mathrm{TM}}$ NCC Oncopanel System (Sysmex Corporation), which facilitates the identification of variants of 114 cancer-related genes (Table 1) via next-generation sequencing (NGS) [7]. This gene panel test included SMARCA4 and SMARCB1, but not SMARCA2. No genomic alterations were found in the 114 genes included in the panel. Thus, he was diagnosed with SMARCA2/BRM-deficient undifferentiated/rhabdoid carcinoma of an unknown primary site with multiple bone 
Table 1. Genes examined using the NCC Oncopanel test

\begin{tabular}{|c|c|c|c|c|c|}
\hline \multicolumn{5}{|c|}{ Mutations and copy number alterations for all exons } & \multirow{2}{*}{$\begin{array}{l}\text { Fusions } \\
\text { ALK }\end{array}$} \\
\hline ABL1 & CRKL & IDH2 & NF1 & RAC2 & \\
\hline ACTN4 & CREBBP & IGF1R & NFE2L2/Nrf2 & RAD51C & AKT2 \\
\hline AKT1 & CTNNB1 & IGF2 & NOTCH1 & RAF1/CRAF & BRAF \\
\hline AKT2 & CUL3 & IL7R & NOTCH2 & RB1 & ERBB4 \\
\hline AKT3 & DDR2 & JAK1 & NOTCH3 & RET & FGFR2 \\
\hline ALK & EGFR & JAK2 & NRAS & RHOA & FGFR3 \\
\hline APC & ENO1 & JAK3 & NRG1 & ROS1 & NRG1 \\
\hline ARAF & EP300 & KDM6A/UTX & NTRK1 & SETBP1 & NTRK1 \\
\hline ARID1A & ERBB2/HER2 & KEAP1 & NTRK2 & SETD2 & NTRK2 \\
\hline ARID2 & ERBB3 & KIT & NTRK3 & SMAD4 & PDGFRA \\
\hline ATM & ERBB4 & KRAS & NT5C2 & SMARCA4/BRG1 & RET \\
\hline AXIN1 & ESR1/ER & MAP2K1/MEK1 & PALB2 & SMARCB1 & ROS1 \\
\hline AXL & EZH2 & MAP2K2/MEK2 & PBRM1 & SMO & \\
\hline BAP1 & FBXW7 & MAP2K4 & PDGFRA & STAT3 & \\
\hline BARD1 & FGFR1 & MAP3K1 & PDGFRB & STK11/LKB1 & \\
\hline BCL2L11/BIM & FGFR2 & MAP3K4 & PIK3CA & TP53 & \\
\hline BRAF & FGFR3 & MDM2 & PIK3R1 & TSC1 & \\
\hline BRCA1 & FGFR4 & MDM4 & PIK3R2 & VHL & \\
\hline BRCA2 & FLT3 & MET & POLD1 & & \\
\hline CCND1 & GNA11 & MLH1 & POLE & & \\
\hline CD274/PD-L1 & GNAQ & MTOR & PRKCl & & \\
\hline CDK4 & GNAS & MSH2 & PTCH1 & & \\
\hline CDKN2A & HRAS & MYC & PTEN & & \\
\hline CHEK2 & IDH1 & MYCN & RAC1 & & \\
\hline
\end{tabular}

metastases and intraperitoneal lymph node metastasis. To alleviate the symptom of cord compression, we performed radiation therapy to treat the thoracic and lumbar spine metastasis (30 Gy in 10 daily fractions of $3 \mathrm{~Gy}$ ) before chemotherapy and left femur metastasis to prevent bone fracture (39 Gy in 13 daily fractions of $3 \mathrm{~Gy}$ ) after about 1-month of the first visit to our hospital. We began administering a monthly subcutaneous injection of denosumab and selected carboplatin (CBDCA) and paclitaxel (CBDCA area under the curve [AUC] 5 on day 1 plus paclitaxel $175 \mathrm{mg} / \mathrm{m}^{2}$ on day 1 every 3 weeks) as first-line chemotherapy after 3 weeks of radiation therapy. Owing to thrombocytopenia, CBDCA was reduced to an AUC of 4 . The regimen was stopped due to an anaphylactic shock occurring immediately after paclitaxel administration. We selected a CBDCA and gemcitabine (GEM) regimen (CBDCA AUC 4 on day 1 plus GEM $750 \mathrm{mg} / \mathrm{m}^{2}$ on day 1 every 3 weeks) as the second chemotherapy regimen. However, the patient did not continuously receive this regimen due to the recovery delay from neutropenia and thrombocytopenia. After 11 weeks of two courses of chemotherapy, CT revealed that the multiple bone metastases increased and enlarged, except for the irradiated lesion and the presence of brain, right axillary lymph nodes, and mediastinal lymph node metastases. The irradiated spine lesions showed osteoblastic bone formation (shown in Fig. 3). Due to the poor response to chemotherapy, the patient died of disease progression approximately 6 months after initiating first-line chemotherapy. 

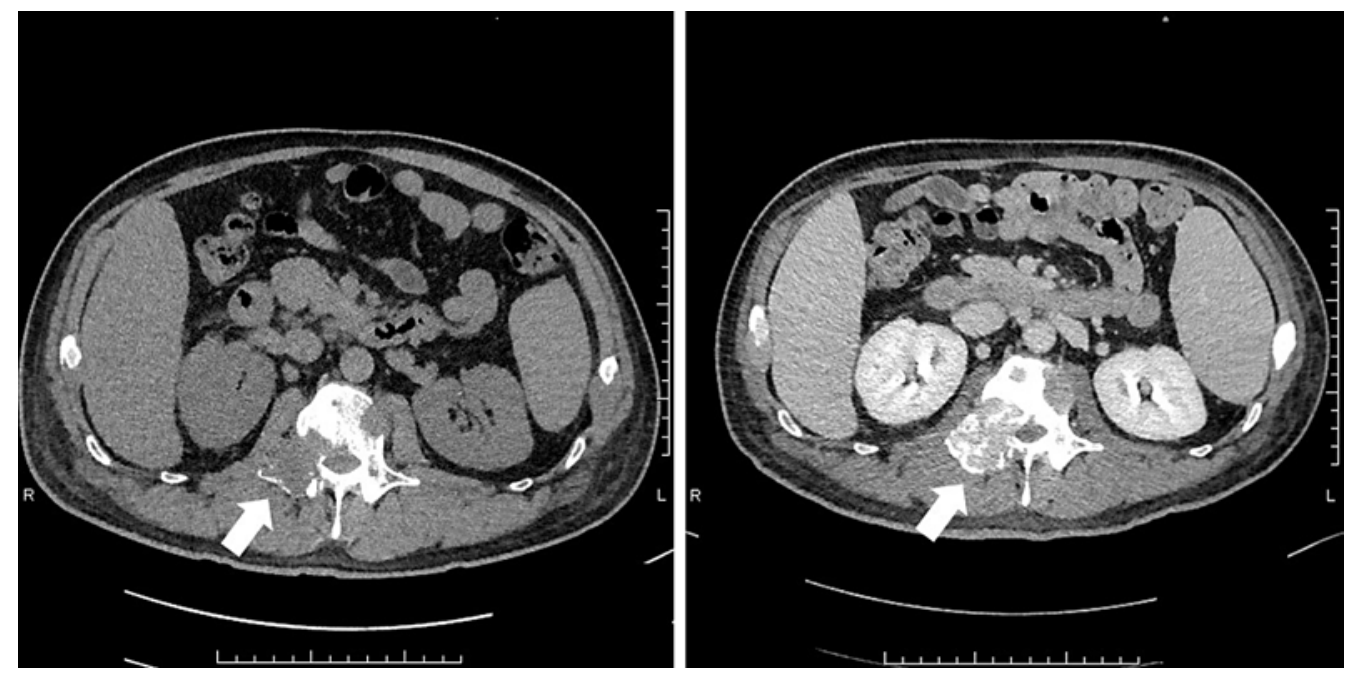

Fig. 3. The osteolytic lesion at the lumbar spine improved after radiation therapy.

\section{Discussion/Conclusion}

To our knowledge, this is the first report on SMARCA2/BRM-deficient undifferentiated/ rhabdoid carcinoma of an unknown primary site. Similar to the present case, SMARCA4-deficient thoracic sarcoma is also frequently deficient in SMARCA2 [4], has an undifferentiated rhabdoid morphology [8], and has a poor prognosis [4]. Deleterious mutations of the SWI/SNF complex indicate tumor-suppressive function loss [9]. Tumors with SWI/SNF deficiency and undifferentiated rhabdoid morphology include small-cell carcinoma of the ovary hypercalcemic type and malignant rhabdoid tumor. Tumors with SWI/SNF deficiency and undifferentiated rhabdoid morphology have also been reported in the uterus [10] and gastrointestinal tract [11]. Tumors with reported SMARCA2 deficiency include non-small-cell lung cancer, prostate cancer, and gastric cancer [3]. Inactivating mutations in several SWI/SNF subunits have recently been frequently identified in various cancers. The importance of altered levels and/or aberrant function of SWI/SNF subunits in carcinogenesis is not fully understood. Approximately $15 \%$ of all cancers display numerous aberrations in SMARCA2 abundance or impairment, which may lead to cancer development or progression [12]. The relationship between SWI/SNF deficiency and undifferentiated rhabdoid morphology is unclear. SMARCA2-deficient undifferentiated/rhabdoid carcinoma was previously only seen in the gallbladder [13]. This reported case had a highly aggressive course, similar to that of our case.

Although the efficacy of the CBDCA and GEM regimens could not be assessed due to the low intensity of the treatment for myelosuppression in our case, both SMARCA2/BRM-deficient undifferentiated/rhabdoid carcinoma and SMARCA4-deficient thoracic sarcoma may be chemoresistant. SMARCA4-deficient thoracic sarcoma is refractory to many kinds of chemotherapy regimens, except for a transient response to Adriamycin-ifosfamide treatment in 1 case [4]. Presently, radiation therapy is very effective in preventing spinal cord compression and relieving lumbar pain. Thus, radiation therapy for symptom relief may be an effective treatment option.

Standard treatment for undifferentiated carcinoma of an unknown primary site has not yet been established. Some clinical studies on CUP have demonstrated the efficacy of combination chemotherapy, such as CBDCA and paclitaxel [14]. However, the results were unsatisfactory with poor overall survival of approximately 1 year, and we should improve the clinical outcome in CUP. A recent phase II study revealed that site-specific and targeted therapy based 
on molecular profiling by NGS for CUP might improve clinical outcomes [15]. It is highly expected that treatment strategies for undifferentiated carcinomas of unknown primary sites will be decided based on the genomic findings of comprehensive genomic profiling to assess the primary site and explore possible targeted therapeutic agents. In addition, the association between genetic alterations and pathological findings will benefit the classification of CUP cases.

It has not been well known that undifferentiated carcinoma of the unknown primary site may include SWI/SNF-deficient tumor. SWI/SNF-deficient tumor is undifferentiated/rhabdoid morphology, aggressive clinical course, and refractory to the platinum agent. Thus, identifying this tumor from undifferentiated carcinoma of an unknown primary site is very important. Immunostaining for SMARCA4, SMARCA2, and SMARCB1 may be useful for diagnosing SWI/ SNF-deficient tumors and the decision of clinical management, if the histopathological findings show undifferentiated/rhabdoid cells. We cannot control the progression of SWI/SNF tumors with the chemotherapy of the platinum doublet regimen. We expect the palliative effect of symptoms with RT. We should search for suitable molecular target agents with comprehensive genomic profiling or examine the effectiveness of immune therapy. We should collaborate with the pathologists to identify SWI/SNF-deficient tumors from poorly differentiated or undifferentiated carcinoma of the unknown primary site and to accumulate more cases and look for further findings to better establish an effective treatment strategy.

\section{Acknowledgments}

The authors would like to thank Editage (www.editage.com) for English language editing.

\section{Statement of Ethics}

This article does not contain any studies with human participants performed by any of the authors. Our institutional Ethics Committee approved the use of his medical record for this case report. The patient provided written informed consent for the publication of this case report and all accompanying images.

\section{Conflict of Interest Statement}

The authors have no conflicts of interest to declare.

\section{Funding Sources}

This study did not receive any funding in any form.

\section{Author Contributions}

Y.T. wrote the manuscript. Y.T. and A.T. managed the patient while in the ward. K.S., K.S., and Y.Y. provided some advice in clinical management. H.I., K.U., and M.U. were the pathologists who made the diagnosis of this tumor and made slides with histopathology pictures. T.M. and I.T. were the department heads and approved all medical decisions. All the authors read and approved the final manuscript.

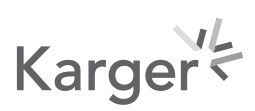




\section{Data Availability Statement}

The data that support the findings of this study are available from the corresponding author (Y.T.) upon reasonable request.

\section{References}

1 Pavlidis N, Fizazi K. Carcinoma of unknown primary (CUP). Crit Rev Oncol Hematol. 2009;69(3):271-8.

2 Schaefer IM, Hornick JL. SWI/SNF complex-deficient soft tissue neoplasms: an update. Semin Diagn Pathol. 2021; 38(3):222-31.

3 Oike T, Ogiwara H, Nakano T, Yokota J, Kohno T. Inactivating mutations in SWI/SNF chromatin remodeling genes in human cancer. Jpn J Clin Oncol. 2013;43(9):849-55.

4 Yoshida A, Kobayashi E, Kubo T, Kodaira M, Motoi T, Motoi N, et al. Clinicopathological and molecular characterization of SMARCA4-deficient thoracic sarcomas with comparison to potentially related entities. Mod Pathol. 2017;30(6):797-809.

5 Lu B, Shi H. An in-depth look at small cell carcinoma of the ovary, hypercalcemic type (SCCOHT): clinical implications from recent molecular findings. J Cancer. 2019;10(1):223-37.

6 Geller JI, Roth JJ, Biegel JA. Biology and treatment of rhabdoid tumor. Crit Rev Oncog. 2015;20(3-4):199-216.

7 Sunami K, Ichikawa H, Kubo T, Kato M, Fujiwara Y, Shimomura A, et al. Feasibility and utility of a panel testing for 114 cancer-associated genes in a clinical setting: a hospital-based study. Cancer Sci. 2019;110(4):1480-90.

8 Sauter JL, Graham RP, Larsen BT, Jenkins SM, Roden AC, Boland JM. SMARCA4-deficient thoracic sarcoma: a distinctive clinicopathological entity with undifferentiated rhabdoid morphology and aggressive behavior. Mod Pathol. 2017;30(10):1422-32.

9 Wilson BG, Roberts CW. SWI/SNF nucleosome remodellers and cancer. Nat Rev Cancer. 2011;11(7):481-92.

10 Strehl JD, Wachter DL, Fiedler J, Heimerl E, Beckmann MW, Hartmann A, et al. Pattern of SMARCB1 (INI1) and SMARCA4 (BRG1) in poorly differentiated endometrioid adenocarcinoma of the uterus: analysis of a series with emphasis on a novel SMARCA4-deficient dedifferentiated rhabdoid variant. Ann Diagn Pathol. 2015; 19(4):198-202.

11 Agaimy A, Daum O, Märkl B, Lichtmannegger I, Michal M, Hartmann A. SWI/SNF complex-deficient undifferentiated/rhabdoid carcinomas of the gastrointestinal tract: a series of 13 cases highlighting mutually exclusive loss of SMARCA4 and SMARCA2 and frequent co-inactivation of SMARCB1 and SMARCA2. Am J Surg Pathol. 2016;40(4):544-53.

12 Jancewicz I, Siedlecki JA, Sarnowski TJ, Sarnowska E. BRM: the core ATPase subunit of SWI/SNF chromatinremodelling complex-a tumour suppressor or tumour-promoting factor? Epigenetics Chromatin. 2019;12(1): 68.

13 Gerber TS, Agaimy A, Hartmann A, Habekost M, Roth W, Stenzinger A, et al. SWI/SNF-deficient undifferentiated/ rhabdoid carcinoma of the gallbladder carrying a POLE mutation in a 30-year-old woman: a case report. Diagn Pathol. 2021;16(1):52.

14 Briasoulis E, Kalofonos H, Bafaloukos D, Samantas E, Fountzilas G, Xiros N, et al. Carboplatin plus paclitaxel in unknown primary carcinoma: a phase II Hellenic Cooperative Oncology Group Study. J Clin Oncol. 2000; 18(17):3101-7.

15 Hayashi H, Takiguchi Y, Minami H, Akiyoshi K, Segawa Y, Ueda H, et al. Site-specific and targeted therapy based on molecular profiling by next-generation sequencing for cancer of unknown primary site: a nonrandomized phase 2 clinical trial. JAMA Oncol. 2020;6(12):1931-8.

\section{Karger'₹}

